\title{
Aase Case of Severe Cerebral Ischemia after Coil Embolization Hemorrhagic Vertebral Artery Dissecting Aneurysm with PICA Originated from V3 Segment
}

Maki Inaba, ${ }^{1}$ Junta Moroi, ${ }^{1}$ and Michihiro Tanaka ${ }^{2}$

Objective: Treatment for ruptured dissecting aneurysms of the vertebral artery (VA) varies according to the origin of the posterior inferior cerebellar artery (PICA). However, a PICA originating from the V3 segment has not been reported.

Case Presentations: A 49-year-old man with a World Federation of Neurosurgical Societies (WFNS) grade III subarachnoid hemorrhage developed headache and bilateral lower limb weakness. Computed tomographic angiography revealed a right hemorrhagic dissecting aneurysm of the VA at the V4 segment, with a PICA originating from the V3 segment. On the day after endovascular coil embolization of the parent artery, magnetic resonance imaging (MRI) identified ischemic complications of the lateral medullary, ipsilateral cerebellar hemisphere. He had no other complications and was transferred to a rehabilitation hospital with modified Rankin scale (mRS)4.

Conclusion: Considering the origins of the PICA and anterior spinal artery (ASA) is important when selecting treatment for hemorrhagic vertebral artery dissecting aneurysm. The morphological features of aneurysms, such as that described herein, are challenging, and perforated vessels might become occluded and initiate ischemic complications.

Keywords > extradural origin PICA, dissecting aneurysm, vertebral artery

\section{Introduction}

The posterior inferior cerebellar artery (PICA) varies. The extra-cranial origin-type PICA is not rare. Strategies to treat dissecting aneurysms of the vertebral artery depend on the position of PICA branching, but no study has reported dissecting aneurysms of the vertebral artery in extra-cranial origin-type PICA patients. In this report, we report an extra-cranial origin-type PICA patient with a dissecting vertebral aneurysm who developed subarachnoid hemorrhage and in whom the underestimation of the PICA perfusion area led to an extensive ischemic complication, and review the literature regarding this issue.

${ }^{1}$ Department of Neurosurgery, Research Institute for Brain and Blood Vessels-Akita, Japan

${ }^{2}$ Department of Neurosurgery, Kameda Medical Center, Kamogawa, Chiba, Japan

Received: October 30, 2015; Accepted: February 20, 2016 Corresponding author: Michihiro Tanaka. Department of Neurosurgery, Kameda Medical Center, 929 Higashi-cho, Kamogawa City, Chiba 296-8602, Japan

Email: dandy25@hotmail.co.jp

(C)2016 The Editorial Committee of Journal of Neuroendovascular

Therapy. All rights reserved.

\section{Case Presentation}

A 49-year-old male.

Medical history: Hypertension, atopic dermatitis.

Present illness: He complained of weakness of the lower limbs during bathing, and requested his family to call an ambulance. Under a diagnosis of subarachnoid hemorrhage made based on imaging findings at a local clinic, he was referred to our hospital.

Physical findings on admission: The Japan Coma Scale score was 10, and the Glasgow Coma Scale score was 13 (E3V4M6), suggesting consciousness disorder. Nuchal rigidity involving a 6-cm area was present. Concerning neurological findings, Manual Muscle Testing (MMT) of the bilateral lower limbs showed 2/5.

Imaging findings on admission: Computed tomography (CT) revealed diffuse subarachnoid hemorrhage involving the basilar cistern (Figs. 1A and 1B). According to criteria established by the World Federation of Neurosurgical Societies, the grade was evaluated as III. It was assessed as 2 in accordance with the Hunt \& Kosnik classification and Group 2 using Fisher's classification. Three-dimensional (3D)-computed tomography arteriography (CTA) showed a fusiform aneurysm, measuring $5.6 \mathrm{~mm}$ in maximum 


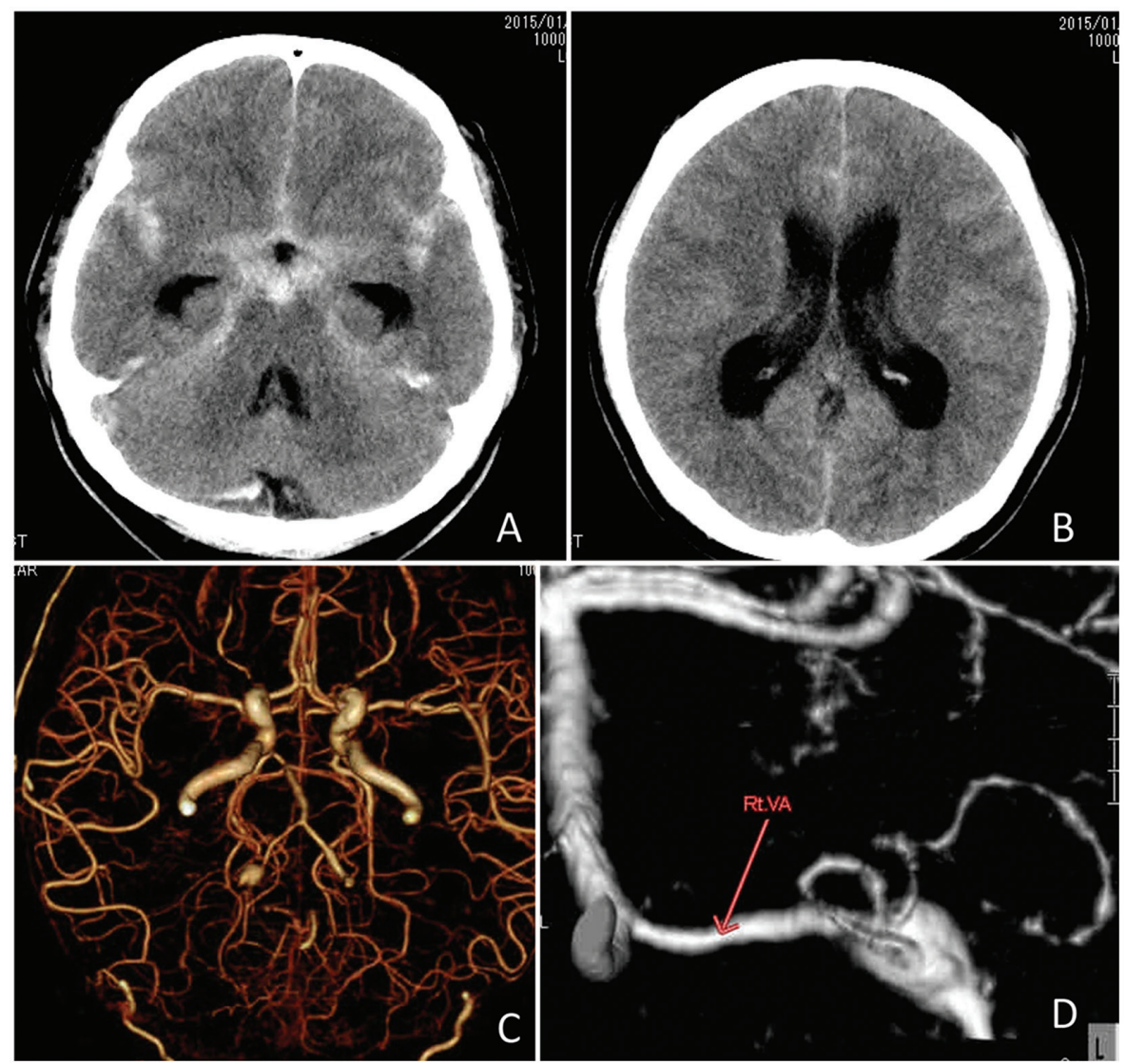

Fig. 1 (A, B) Axial unenhanced CT shows diffuse thick subarachnoid hemorrhage. (C) CTA shows rather dominant left vertebral artery. (D) A three-dimensional angiogram of the right vertebral artery. Dissecting aneurysm with 2 perforators (arrow), originating from the wall of the aneurysm. CT: computed tomography; CTA: computed tomography arteriography

diameter, in the V4 portion of the right vertebral artery, and two branches were originated from the aneurysm (Figs. 1C and 1D). There was no anterior inferior cerebellar artery (AICA). On cerebral angiography, the diameter of the left vertebral artery was greater than that of the right vertebral artery, and the bilateral PICAs were originated from the extra-cranial region (Figs. 2A and 2B).

Treatment course: Cerebral angiography led to a diagnosis of a ruptured dissecting aneurysm of the vertebral artery. The diameter of the left vertebral artery was greater than that of the right vertebral artery, and the PICAs were originated from the extra-cranial region, being distant from the site of dissection. As there was no AICA, we could not rule out the possibility that a blood vessel directly branching from the aneurysm may involve the AICA region, but embolization was selected to achieve hemostasis, considering that the patient developed subarachnoid hemorrhage (Fig. 2C). Bypass was not selected for the following reasons: anastomosis to a blood vessel directly branching from the aneurysm is difficult, and stenting may lead to incomplete hemostasis.
Intraoperative findings: Under general anesthesia, a 6Fr long sheath was inserted into the right femoral artery. As a diagnostic catheter for the left vertebral artery, a 4Fr long sheath was inserted into the left femoral artery. A 6Fr Guiding Catheter (Lancher, $90 \mathrm{~cm} \mathrm{STR}$ ) was co-axially combined with a 4Fr CX catheter and 0.035 -inch radifocus guide wire through the right femoral artery, and inserted into the right vertebral artery. Using a microguidewire (Silverspeed, 0.014 inch, $200 \mathrm{~cm}$ ) and microguidecatheter (SL10, $45^{\circ}$ preshaped), it was guided to the distal dissection site. However, stenosis was present at this site, and cannulation was slightly difficult (Fig. 2D). A Target $360 \mathrm{soft}$ coil measuring $3 \mathrm{~mm} \times 8 \mathrm{~cm}$ was inserted through the distal dissection site. For embolization, a total of nine coils were placed from the dissecting space to an area $5 \mathrm{~mm}$ on the proximal side of dissection (Fig. 2E). Partially, the coil slid down into the dissecting space with kick-back motions, but it was detached. Final angiography showed blockage of anterograde blood flow from the right vertebral artery and congestion of blood flow between the embolic site and PICA (Figs. 3A and 3B). Left 


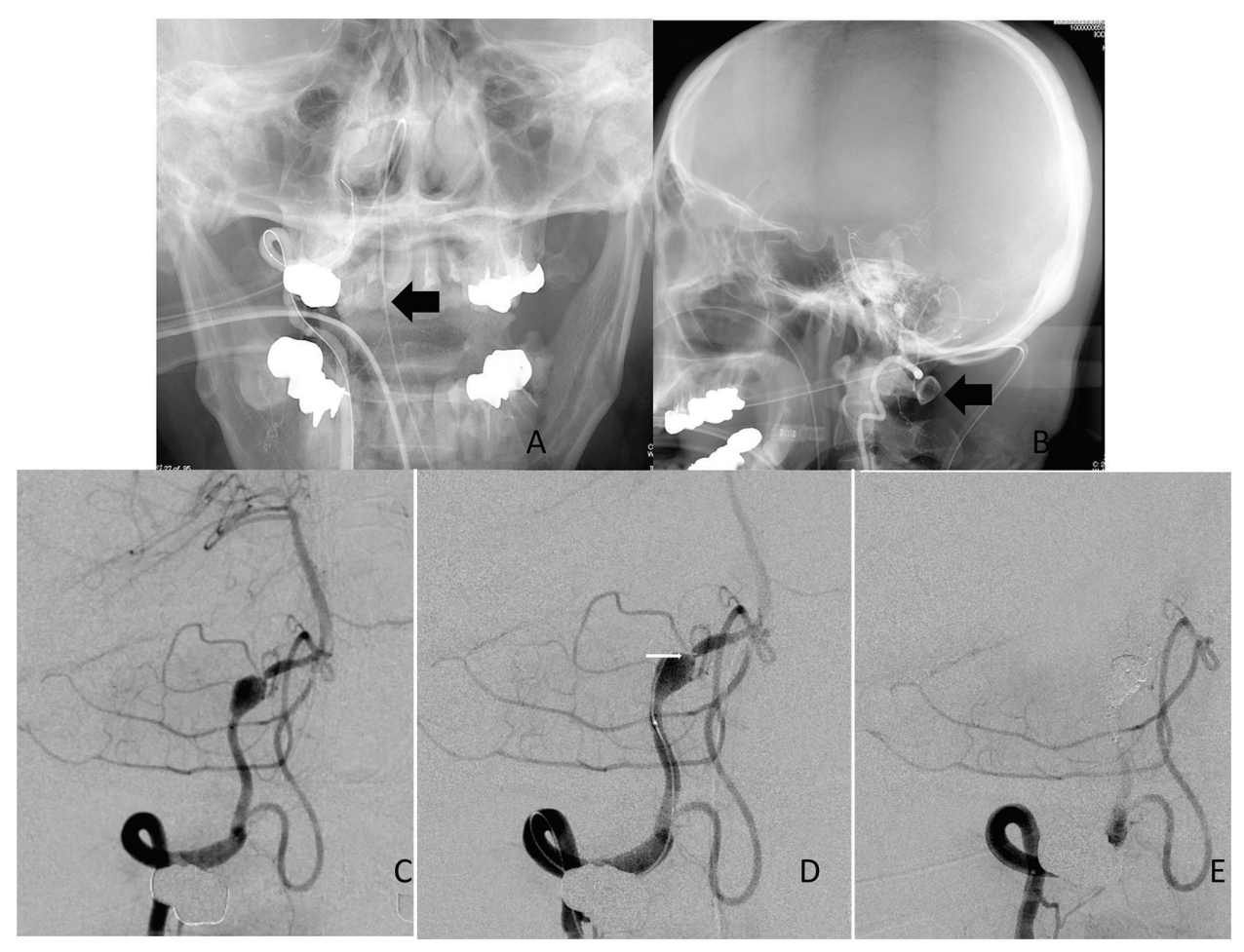

Fig. 2 (A, B) Angiogram with bone of skull shows extracranial origin PICA (arrow). (C) Angiogram (anteroposterior view) of the right vertebral artery shows dissecting aneurysm with irregular wall and perforators which runs off cerebellar hemisphere. There is no anterior inferior cerebellar artery. (D) An intraoperative image shows the tips of a microcatheter (arrow) in the dissecting aneurysm. (E) Angiogram after coil embolization revealed obliteration of the aneurysm, and just the proximal part of the right vertebral artery. PICA: posterior inferior cerebellar artery

vertebral arteriography revealed blockage of blood flow at the distal dissection site of the right vertebral artery and congestion of blood flow at the cecum. After confirming occlusion of the dissection, this procedure was completed (Figs. 3C and 3D). During surgery, heparin was administered so that the activating clotting time (ACT) was prolonged to a value twice higher than the control value. At the completion of surgery, heparin was naturally reversed, and the patient was admitted to the Intensive Care Unit under general anesthesia and intubation management.

Postoperative course: On the 1st postoperative day, diffusionweighted images (DWIs) on magnetic resonance imaging (MRI) after the completion of sedation showed extensive infarction involving the right lateral medulla oblongata and right cerebellar hemisphere. Infarction was partially present at the margin of the left cerebellar hemisphere (Fig. 4). At this point, the Japan coma scale (JCS) score was 30 , and the Glasgow coma scale (GCS) score was E2VTM4. Paralysis of the bilateral limbs (MMT: 2/5), which was possibly related to an increase in the cerebral pressure, was observed, and emergency ventricular drainage was performed. On the 3 rd postoperative day, the JCS and GCS scores were 3 and E4VTM6, respectively, and limb paralysis reduced to an MMT score of $3 / 5$, making it possible for the patient to accept instructions. So, we tried to perform extubation, but it was difficult to excrete sputum, leading to dyspnea. Additional intubation was conducted. On the 6th postoperative day, tracheotomy was performed. In the spasm phase, there was no new neurological deficit, and paralysis of the limbs reduced to an MMT score of 4/5. However, dysphagia and cerebellar symptoms became marked with an improvement in awaking. On the 55th postoperative day, chronic rehabilitation was introduced (mRS4). At this point, the JCS and GCS scores were 3 and E4VTM6, respectively, and proximal muscle-predominant paralysis of the limbs was noted (MMT: 4/5). The MMT score of the distal muscle was 5/5. Thermohypoesthesia of the right face and left upper/lower limbs, dysphagia, and ataxic gait/vertigo persisted, but voice training was conducted under tracheotomy. The patient could communicate with others using a personal computer. Subsequently, hearing impairment of the right ear was noted on wearing ear phones in the Rehabilitation Ward. An auditory brainstem response (ABR) test showed latency prolongation (3-5 waves). Antiplatelet therapy with cilostazol at $200 \mathrm{mg}$ was started on the 1st postoperative day. 


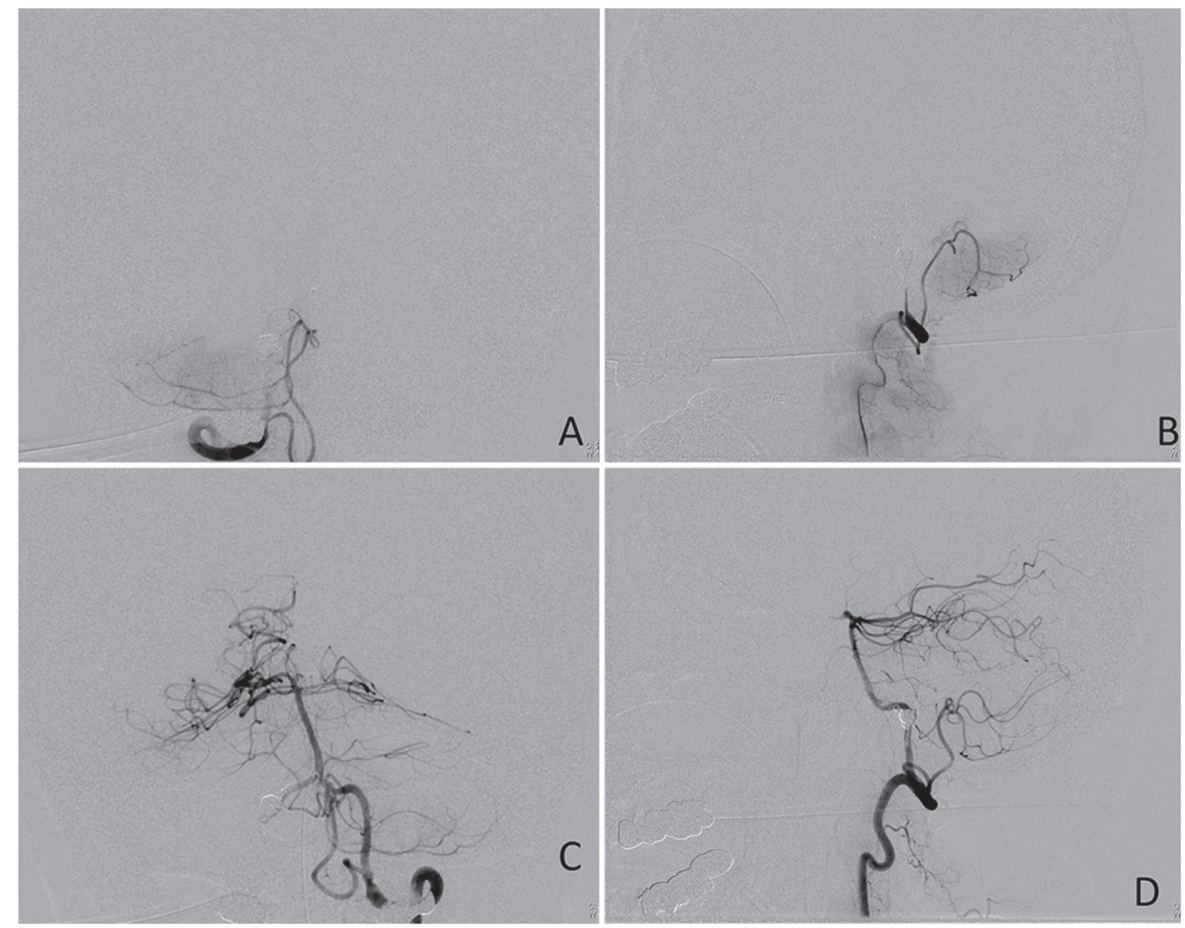

Fig. 3 (A) Right vertebral angiogram after parent artery occlusion (anteroposterior view). No aneurysmal sac was shown on right vertebral angiogram. (B) Right vertebral angiogram after parent artery occlusion (lateral view). (C) Left vertebral angiogram after parent artery occlusion (anteroposterior view). No aneurysmal sac was shown on the left vertebral angiogram. Contrast medium was stagnated distal to the aneurysm, and no contrast was seen in the right lateral cerebellar hemisphere. (D) Left vertebral angiogram after parent artery occlusion (lateral view).
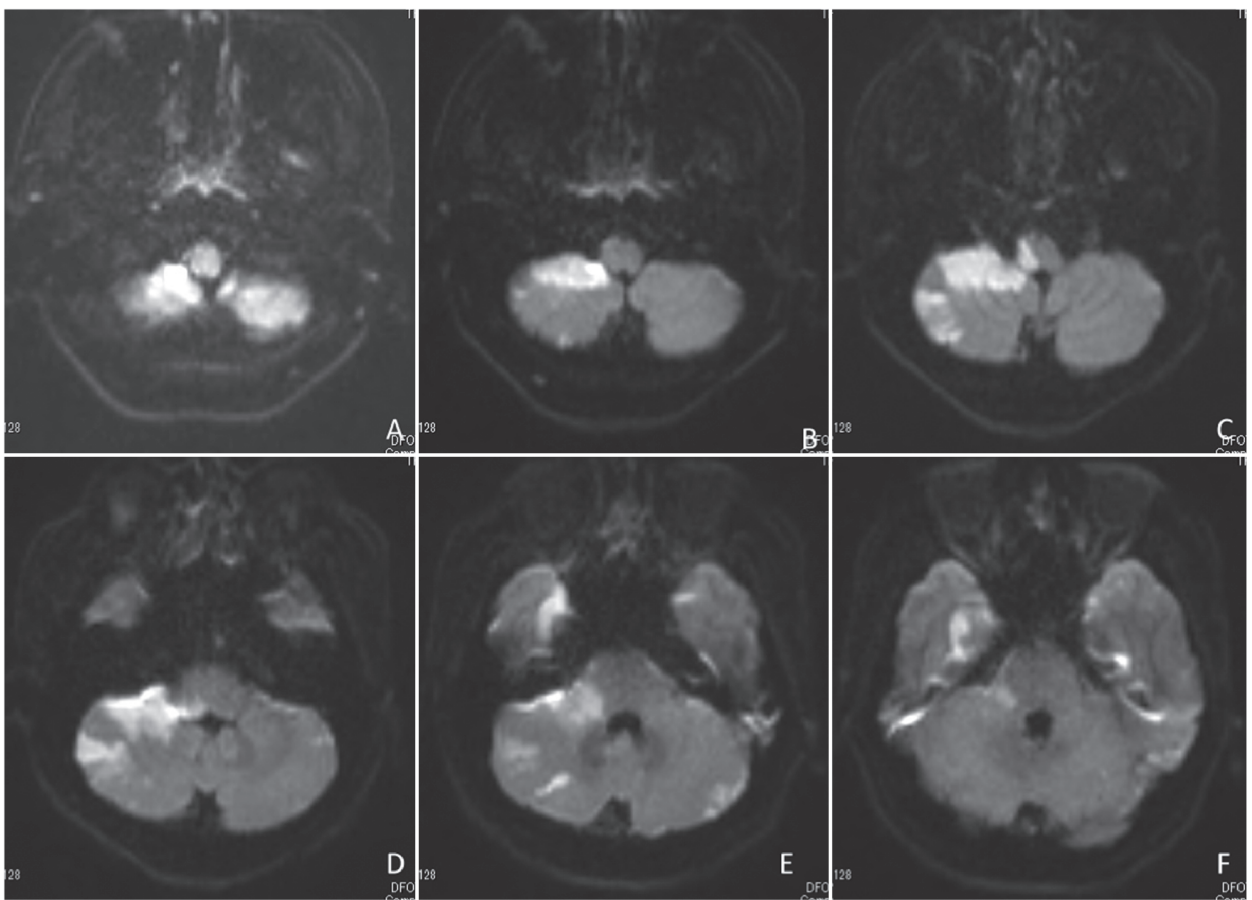

Fig. 4 A Diffusion-weighted MR image reveals some high-intensity lesions mainly on the inferior olivery nucleus, lateral medulla, lateral pons, middle cerebellar peduncle on the right side, inferior cerebellar hemisphere on both sides. MR: magnetic resonance 


\section{Discussion}

With respect to strategies to treat hemorrhagic dissecting aneurysms of the vertebral artery, it is described that the incidence of additional hemorrhage ranges from 14 to $69 \%$, and that surgery/endovascular treatment to prevent additional hemorrhage must be considered in the Japanese Guidelines for the Management of Stroke 2015. However, recently, endovascular treatment has been increasingly selected as a first-choice treatment. For treatment at this site, it is recommended that treatment should be selected based on the type of the bifurcation point between the vertebral artery (VA) and PICA: pre-PICA, PICA-involved, post-PICA, or nonPICA types. In pre-PICA- and non-PICA-type patients, the VA involving the site of dissection should be embolized. The PICA on the affected side should be retrogradely preserved. In post-PICA-type patients, the VA must be embolized between the site of dissection and PICA. This is difficult if the distance between the bifurcation point of the PICA and site of dissection is short in PICA-involved- or post-PICAtype patients. Bypass should be performed in the affectedside PICA region, or, if the affected-side PICA is not predominant, there is another option: the site of dissection involving the PICA is embolized, considering the PICA collateral pathway. When there is no resistance to ischemia, stenting may be considered, but intracranial stenting has not been approved in Japan. Some studies indicated that the use of a stent facilitated the preservation of a penetrating vessel area, whereas others reported additional hemorrhage; a consensus has not been reached. ${ }^{1-8)}$

The origin of the PICA, which is important for determining therapeutic strategies, varies. The extradural-origin-type PICA is not rare, and its detection rate reportedly ranges from approximately 5 to $20 \%{ }^{9-13)}$ Many studies have investigated intracranial-origin-type PICAs, such as duplicate and aplastic PICAs. However, only some case reports on extraduralorigin-type PICAs have been published, and no study has examined penetrating-vessel infarction. According to Lister et al., the PICA is classified into five segments: anterior medullary, lateral medullary, tosilomedullary, telovelotonsiller, and cortical segments. This classification is based on the anatomical position and curvature, differing from that of arteries on the tent; therefore, it is conveniently used for extraduralorigin-type PICA patients, but it is useful for estimating branch/perfusion areas. The anterior medullary segment branches from the VA, reaching the inferior olive of the lateral medulla oblongata through the anterior inferior margin of the medulla oblongata to the 12th nerve root. The lateral medullary segment involves the inferior olive of the lateral medulla oblongata to the 9th, 10th, and 11th cranial nerve roots. The tosilomedullary segment shows a superior directional change in an area posterior to the 9th, 10th, and 11th cranial nerves at the posterior margin of the medulla oblongata, ascending along the posterior wall of the 4th ventricle and reaching the cerebellar supratonsiller pole. The telovelotonsiller segment involves the medial cerebellar tonsil, branching to the vermis, tonsil, and cortex toward the superior margin of the 4th ventricle. The cortical segment branches into the cortex, reaching the periphery. ${ }^{14,15)}$

The course of the extradural-origin-type PICA involves the extra-cranial area to posterior fossa, reaching the vermis and inferior cerebellar hemisphere through the dorsal medulla oblongata from a site corresponding to the tosilomedullary segment. Therefore, a perfusion area corresponding to the anterior and lateral medullary segments originates from other arteries, especially the VA in most cases. If AICA development is favorable, perfusion from the AICA to the PICA may be achieved, but, when the AICA does not branch, blood may be primarily perfused from VA branches. According to Rhoton et al., branching to the brainstem occurs prior to the anterior medullary, lateral medullary, and tonsilomedullary segments of the PICA. In the tonsilomedullary segment, the largest number of penetrating vessels branch. Branches from the former half of this segment mutually form a complex network. Akar et al. indicated that main perforators to the brainstem branched from the lateral medullary segment and former half of the tonsilomedullary segment based on cadaver results. Furthermore, they reported that arteries perfusing to the medulla oblongata consisted of the VA, anterior spinal artery, AICA, and PICA based on the results of cadaver dissection in 11 patients, and classified branches to the pyramid and olive on the ventral side of the medulla oblongata. According to this classification, perfusion to the pyramid is originated from the anterior spinal artery in many cases, and the inferior olive is present in the PICA perfusion area. Branches from the lateral medullary segment are responsible for this area. ${ }^{15-17)}$

Considering these results of cadaver dissection and findings of infarction in the present case, the blood vessel branching from the site of VA dissection in the present case may have involved an area corresponding to the lateral medullary segment of the PICA. As there was no AICA, this vessel may also have been responsible for the AICA perfusion area. Usually, the VA, AICA, and PICA comprise a complex network; therefore, severe infarction may not occur in the posterior fossa. However, in the present case, the loss of 
VA/AICA/PICA blood flow was suggested. This may have led to infarction of the cerebellar cortex and an extensive area as an area corresponding to the lateral medullary segment and the AICA perfusion area.

On admission, this patient complained of weakness of the bilateral lower limbs, which could not be explained by a hemorrhagic dissecting aneurysm of the right vertebral artery alone. Concerning this, subarachnoid hemorrhage in a relatively young patient was possibly related to an increase in the intracranial pressure. After surgery, consciousness disorder was protracted, which was possibly associated with an increase in the intracranial pressure, and ventricular drainage was performed, gradually reducing the symptoms.

With respect to anticoagulant and antiplatelet therapies in the present case, intraoperative heparin therapy was naturally reversed, and the administration of cilostazol, as an antiplatelet drug, was started the day after surgery. Although a consensus regarding postoperative anticoagulant and antiplatelet therapies for hemorrhagic dissecting aneurysms of the vertebral artery has not been reached, the oral administration of an antiplatelet drug is started after surgery in many cases according to case reports. Concerning treatment immediately after surgery, Sugiu et al. routinely used heparin at a gradually decreased dose and sodium ozagrel, and reported favorable results. $2,3,8,18,19)$

In the present case, a hemorrhagic dissecting aneurysm of the VA, involving a medullary perforator of the VA, with an extradural-origin-type PICA was detected. Concerning treatment, coil embolization was selected for the following reasons: (1) the PICA was originated from the extra-cranial area, being distant from the dissected space, (2) the visualization of the left VA was favorable, suggesting the predominance of the left side, and (3) it was controversial whether or not the concurrent use of a stent leads to complete hemostasis for the ruptured aneurysm. The first reason was a mistake, and it was necessary to regard the bifurcation point between the VA and PICA as PICA-involved type. As a result, serious cerebral infarction occurred, but the prevention of additional aneurysmal rupture was successful.

\section{Conclusion}

(1) We encountered a patient with an ischemic complication after parent artery occlusion (PAO) of the extraduralorigin-type PICA. (2) Assuming that the extradural-origintype PICA perfusion area is extensive, embolization involving perforators branching from the aneurysm was performed, and an extensive infracted focus appeared. (3) When selecting strategies to treat dissecting aneurysms of the vertebral artery with an extradural-origin-type PICA, an intra-dural PICA perfusion area must be considered.

\section{Conflict of Interest}

There is no conflict of interest for the author and coauthors.

\section{References}

1) Mizutani $T$, Aruga $T$, Kirino $T$, et al: Recurrent subarachnoid hemorrhage from untreated ruptured vertebrobasilar dissecting aneurysms. Neurosurgery 1995; 36: 905-911; discussion 912-913.

2) Sugiu K, Tokunaga K, Watanabe K, et al: Endovascular treatment of ruptured vertebral artery dissecting aneurysms: its usefulness and lessons from complications. Surgery for Cerebral Stroke 2005; 33: 200-205. (in Japanese)

3) Sugiu K, Tokunaga K, Date I: Endovascular treatment for ruptured vertebral artery dissecting aneurysms. Neurological Surgery 2004; 32: 1229-1238. (in Japanese)

4) Yoshida K, Oishi H, Yamamoto M, et al: A case of hemorrhagic vertebral artery dissecting aneurysm treated with sole stenting technique. JNET 2015; 9: 103-107. (in Japanese)

5) Narata AP, Yilmaz H, Schaller K, et al: Flow-diverting stent for ruptured intracranial dissecting aneurysm of vertebral artery. Neurosurgery 2012; 70: 982-988; discussion 988-989.

6) Zenteno MA, Santos-Franco JA, Freitas-Modenesi JM, et al: Use of the sole stenting technique for the management of aneurysms in the posterior circulation in a prospective series of 20 patients. J Neurosurg 2008; 108: 1104-1118.

7) Nakagawa T, Katayama M, Murakami H: Enlargement of a PICA-involved-type vertebral artery dissecting aneurysm after recanalization of an endovascularly occluded vertebral artery. Case Report Surgery for Cerebral Stroke 2009; 37: 461-465. (in Japanese)

8) Miyamoto N, Naito I, Takatama S, et al: Stent-assisted coil embolization for ruptured vertebrobasilar dissecting aneurysms using enterprise VRD. Surgery for Cerebral Stroke 2013; 41: 253-258. (in Japanese)

9) Fine $\mathrm{AD}$, Cardoso A, Rhoton AL: Microsurgical anatomy of the extracranial-extradural origin of the posterior inferior cerebellar artery. J Neurosurg 1999; 91: 645-652.

10) Salas E, Ziyal IM, Bank WO, et al: Extradural origin of the posteroinferior cerebellar artery: an anatomic study with histological and radiographic correlation. Neurosurgery 1998; 42: 1326-1331.

11) Chen CJ, Chen ST: Extracranial distal aneurysm of posterior inferior cerebellar artery. Neuroradiology 1997; 39: 344-347. 
12) Tabatabai SA, Zadeh MZ, Meybodi AT, et al: Extracranial aneurysm of the posterior inferior cerebellar artery with an aberrant origination: case report. Neurosurgery 2007; 61: E1097-E1098; discussion E1098.

13) Tanaka A, Kimura M, Yoshinaga S, et al: Extracranial aneurysm of the posterior inferior cerebellar artery: case report. Neurosurgery 1993; 33: 742-744; discussion 744-745.

14) Lister JR, Rhoton AL, Matsushima $T$, et al: Microsurgical anatomy of the posterior inferior cerebellar artery. Neurosurgery 1982; 10: 170-199.

15) Rodríguez-Hernández A, Rhoton AL, Lawton MT: Segmental anatomy of cerebellar arteries: a proposed nomenclature. Laboratory investigation. J Neurosurg 2011; 115: 387-397.
16) Akar ZC, Dujovny M, Gómez-Tortosa E, et al: Microvascular anatomy of the anterior surface of the medulla oblongata and olive. J Neurosurg 1995; 82: 97-105.

17) Lasjaunias $P$, Vallee $B$, Person $H$, et al: The lateral spinal artery of the upper cervical spinal cord. Anatomy, normal variations, and angiographic aspects. J Neurosurg 1985; 63: 235-241.

18) Yamada NK, Cross DT, Pilgram TK, et al: Effect of antiplatelet therapy on thromboembolic complications of elective coil embolization of cerebral aneurysms. Am J Neuroradiol 2007; 28: $1778-1782$.

19) Halbach VV, Higashida RT, Dowd CF, et al: Endovascular treatment of vertebral artery dissections and pseudoaneurysms. J Neurosurg 1993; 79: 183-191. 